\title{
EDITORIAL
}

\section{More evidence that physical activity is beneficial for prostate}

\section{cancer}

(c) The Author(s), under exclusive licence to Springer Nature Limited 2022

Prostate Cancer and Prostatic Diseases (2022) 25:383-384; https:// doi.org/10.1038/s41391-022-00509-6

There is growing consensus that physical activity is beneficial for men with prostate cancer after diagnosis, with consistent findings observed in multiple large cohort studies over the past 10 years for the outcome of prostate cancer-specific mortality and overall mortality. A recent systematic review and meta-analysis by Friedenreich et al. reported a $30 \%$ risk reduction for prostate cancer mortality and a $40 \%$ risk reduction for all-cause mortality for the most vs. least active prostate cancer survivors [1]. Other data indicate that more vigorous activity may yield further benefits [2]. However, additional studies showing shorter-term clinical benefits would further motivate men to incorporate changes in their physical activity habits.

The report by Brassetti et al. [3] in this issue of Prostate Cancer and Prostatic Diseases evaluated physical activity level with risk of prostate cancer reclassification during active surveillance in 85 patients. At a median follow-up of 37 months, one out of three patients experienced reclassification, and these patients were characterized as less physically active at baseline $(p=0.056)$, while also having smaller prostates $(p=0.001)$ and a higher PSA doubling time at baseline $(p=0.019)$. The study reported reclassification probabilities of 39,21 , and $13 \%$ at 2 yrs and probabilities of $66 \%, 35 \%$, and $13 \%$ at 5 yrs (log rank $p=0.033)$ for those with activity levels classified as sedentary, moderately active, and active, respectively. In a multivariate Cox proportional hazards regression analyses, a continuous measure of physical activity (assessed via the Physical Activity Score for the Elderly (PASE) survey) was slightly inversely associated with risk of reclassification. The difference in median PASE score was 18 points, between those who did not experience reclassification vs. those who did ( 87.8 vs. $69.3, p$ value 0.056 ). PASE score was the only statistically significantly independent predictor of reclassification in the analysis, though PSA density was positively associated with a $p$ value $\sim 0.054$. These reclassification results support previous work in two studies in active surveillance patients showing that a comprehensive lifestyle intervention (which included exercise) reduced the odds of reclassification at 2 yrs [4] and that weekly vigorous activity can also reduce these odds [5].

The results in the current study are striking, though should be interpreted with caution given that the physical activity assessment was subject to recall and social desirability bias, as mentioned by the authors. Nevertheless, if corroborated, the data suggest that men with prostate cancer who are active could defer or even avoid treatment and their adverse side effects, and moreover, combat common cancer-related health outcomes, including anxiety, depression, fatigue, poor physical function, and other health-related quality of life metrics [6]. Unfortunately, very few patients will experience this benefit. In this study, only $18 \%$ of the population was considered active, while $54 \%$ were moderately active, and $28 \%$ were sedentary. These figures align with current trends in the United States where $34 \%$ of cancer survivors aged 18 years and older report no physical activity in their leisure time and only $16 \%$ meet national guidelines for aerobic and muscle-strengthening physical activity [7]. A number of trials have evaluated various exercise intensities and combinations of modalities on a variety of clinical, functional, and psychosocial outcomes, summarized previously [8]. Other recently completed and ongoing studies in prostate cancer are taking a more remote-based approach to exercise training, in part to reduce participant burden and encourage study participation, including the Active Surveillance Exercise Clinical Trial in active surveillance patients (NCT02435472), the CHAMP (Clinical trial of High-intensity Aerobic and resistance exercise for Metastatic castrate-resistant Prostate cancer) study [9], and more recently, the global INTERVAL-GAP4 (INTense ExeRcise for survival among men with Metastatic Prostate Cancer) study [10], the latter which currently allows for sites to provide either onsite or remote supervision. Our team is examining a 4-month remotely supervised aerobic exercise intervention vs. usual care among men with prostate cancer on active surveillance to examine changes in fitness and cancer biomarkers. This trial, slated for completion in 2022, may shed light on biological mechanisms underlying the potential benefit reported herein by Brassetti et al.

Providing exercise guidance as part of the survivorship journey to patients should become integrated as standard of care, as the consensus statement from a recent International Multidisciplinary Roundtable concluded that every survivor should "avoid inactivity" [6]. Finding ways to support patients to increase physical activity and address disparities that create barriers to engagement in physical activity should be critical pillars of a personalized cancer management plan that is offered to the patient during and after treatment.

Some aspects of the study should be noted. The study was retrospective in nature and physical activity was only measured once. It is unknown how these estimates would change with updated physical activity data. The activity survey used did not assess exercise intensity. While vigorous exercise should be recommended if patients can engage in activity at that intensity level, more recent studies suggest that moderate-intensity activity is also beneficial for prostate cancer and overall mortality. Moreover, for patients who are inactive, recommending smaller bouts and shorter duration of activity is a necessary starting place. The NHANES study reported that less-active US adults who replaced $1 \mathrm{~h}$ of sedentary time with either light- or moderate-tovigorous activity had a $18 \%$ and $42 \%$ lower risk of total mortality [11] — so every little bit counts.

Additional studies, like this, are also needed in diverse groups. This retrospective study was completed in an Italian, fully Caucasian cohort; additional efforts must be made, especially in prospective cohorts and randomized controlled trials, to incorporate under-represented prostate cancer patients. 
Overall, the findings of this research are provocative and provide further motivation to 'get off the couch,' and engage in or promote activity. Physical activity is not only beneficial for prostate cancer outcomes, but also for mental health/well-being and cardiovascular and other chronic diseases.

\section{Stacey A. Kenfield (iD) ${ }^{1 凶}$ and June M. Chan ${ }^{1}$ ${ }^{1}$ University of California, San Francisco, San Francisco, CA, USA. 凶email: Stacey.Kenfield@ucsf.edu}

\section{REFERENCES}

1. Friedenreich CM, Stone CR, Cheung WY, Hayes SC. Physical activity and mortality in cancer survivors: a systematic review and meta-analysis. JNCI Cancer Spectr. 2020;4:pkz080.

2. Kenfield SA, Stampfer MJ, Giovannucci E, Chan JM. Physical activity and survival after prostate cancer diagnosis in the Health Professionals Follow-Up Study. J Clin Oncol. 2011;29:726-32.

3. Brassetti A, Ferriero M, Napodano G, Sanseverino R, Badenchini F, Tuderti G, et al. Physical activity decreases the risk of cancer reclassification in patients on active surveillance: a multicenter retrospective study. Prostate Cancer and Prostatic Dis. 2021;24:1151-57.

4. Frattaroli J, Weidner G, Dnistrian AM, Kemp C, Daubenmier JJ, Marlin RO, et al. Clinical events in prostate cancer lifestyle trial: results from two years of followup. Urology. 2008;72:1319-23.

5. Guy DE, Vandersluis A, Klotz LH, Fleshner N, Kiss A, Parker C, et al. Total energy expenditure and vigorous-intensity physical activity are associated with reduced odds of reclassification among men on active surveillance. Prostate Cancer Prostatic Dis. 2018;21:187-95.

6. Campbell KL, Winters-Stone KM, Wiskemann J, May AM, Schwartz AL, Courneya $\mathrm{KS}$, et al. Exercise guidelines for cancer survivors: consensus statement from International Multidisciplinary Roundtable. Med Sci Sports Exerc. 2019;51:2375-90.

7. Centers for Disease Control and Prevention: National Center for Health Statistics. National Health Interview Survey, 1997-2018. https://progressreport.cancer.gov/ after/physical_activity.
8. Zuniga KB, Chan JM, Ryan CJ, Kenfield SA. Diet and lifestyle considerations for patients with prostate cancer. Urol Oncol. 2020;38:105-17.

9. Kenfield SA, Van Blarigan EL, Panchal N, Bang A, Zhang L, Graff RE, et al. Feasibility, safety, and acceptability of a remotely monitored exercise pilot CHAMP: A Clinical trial of High-intensity Aerobic and resistance exercise for Metastatic castrate-resistant Prostate cancer. Cancer Med. 2021;10:8058-70.

10. Newton RU, Kenfield SA, Hart NH, Chan JM, Courneya KS, Catto J, et al. Intense Exercise for Survival among Men with Metastatic Castrate-Resistant Prostate Cancer (INTERVAL-GAP4): a multicentre, randomised, controlled phase III study protocol. BMJ Open. 2018;8:e022899.

11. Matthews CE, Keadle SK, Troiano RP, Kahle L, Koster A, Brychta R, et al. Accelerometer-measured dose-response for physical activity, sedentary time, and mortality in US adults. Am J Clin Nutr. 2016;104:1424-32.

\section{AUTHOR CONTRIBUTIONS}

Writing —original: SAK. Writing — review and editing: SAK and JMC.

\section{COMPETING INTERESTS}

The authors declare no competing interests.

\section{ADDITIONAL INFORMATION}

Correspondence and requests for materials should be addressed to Stacey A. Kenfield.

Reprints and permission information is available at http://www.nature.com/ reprints

Publisher's note Springer Nature remains neutral with regard to jurisdictional claims in published maps and institutional affiliations. 